\title{
Influence of small miscuts on self-ordered growth of Ge nanoislands
}

\author{
O.Yo. Gudymenko ${ }^{1}$, V.P. Kladko ${ }^{1}$, O.M. Yefanov ${ }^{1}$, M.V. Slobodian ${ }^{1}$, Yu.S. Polischuk ${ }^{1}$, \\ Z.F. Krasilnik ${ }^{2}$, D.V. Lobanov ${ }^{2}$, A.A. Novikov ${ }^{2}$ \\ ${ }^{1}$ V. Lashkaryov Institute of Semiconductor Physics, NAS of Ukraine, \\ 45, prospect Nauky, 03028 Kyiv, Ukraine \\ ${ }^{2}$ Institute of Microstructure Physics, RAS, \\ 603600 Nizhny Novgorod, GSP-105, Russia
}

\begin{abstract}
Using high-resolution X-ray diffraction (HRXRD), we have investigated lateral ordering the nanoislands formed from Ge wetting layer of various thicknesses deposited on a strained $\mathrm{Si}_{1-\mathrm{x}} \mathrm{Ge}_{\mathrm{x}}$ sublayer. We observed that the high lateral ordering degree is initiated by ordered modulation of non-uniform deformation fields. This modulation is induced by small $\left(\sim 0.3^{\circ}\right)$ misorientation of $\mathrm{Si}$ substrate from [001] direction. Finally, we show that the miscut can be the source of perfectly ordered nanoisland arrays in two dimensions when the growth is performed on the strained $\mathrm{SiGe}$ (001) sublayer. The effect of substrate miscut can be amplified tuning the deviation of buffer layer surface from [001] growth direction via increasing the Ge content.
\end{abstract}

Keywords: SiGe nanoislands, miscut, deformation field, high-resolution X-ray diffraction.

Manuscript received 20.07.11; revised manuscript received 29.08.11; accepted for publication 14.09.11; published online 30.11.11.

\section{Introduction}

Self-organization is the most perspective method to form nanoislands during epitaxial growth [1]. But it is known that the spontaneous nucleation of nanoislands in accord with the classical Stranski-Krastanov mechanism is accompanied by their chaotic arrangement [2]. Today several techniques are used to obtain ordered arrays of $\mathrm{Ge}(\mathrm{Si})$ nanoislands. In recent years, to organize arrays of $\mathrm{Ge}(\mathrm{Si})$ islands, they use their growth on an undulating surface formed by deposition of $\mathrm{Ge}_{\mathrm{x}} \mathrm{Si}_{1-\mathrm{x}}$ layers [3], selective epitaxial growth of $\mathrm{Si}$ and $\mathrm{Ge}$ in windows and grooves of $\mathrm{SiO}_{2}$ [4], growth on relaxed buffer layers $\mathrm{Si}_{1-\mathrm{x}} \mathrm{Ge}_{\mathrm{x}}$, containing a grid of misfit dislocations [5], the growth in pre-structured $\mathrm{Si}$ substrates [6]. Application of these techniques allows obtaining the ordered chains of nanoislands with a high linear density, but in this case their two-dimensional density still remains low.

The possibility of spontaneous spatial ordering appears with elastic interaction of neighbor nanoislands, when the distance between them is of the order of their sizes in the growth plane $[7,8]$. Reduction of this distance can be achieved by increasing the nanoisland density via decreasing the growth temperature. But it is followed also with reduction of the nanoisland size.

One of the most effective techniques to form dense ordered arrays of $\mathrm{GeSi}$ islands can be their growth on $\mathrm{Ge}_{\mathrm{x}} \mathrm{Si}_{1-\mathrm{x}}$ buffer layers [9, 10]. It is known from [11] that the deposition of strained $\mathrm{SiGe}$ layer on $\mathrm{Si}(001)$ increases the growth plane roughness. This, in turn, leads to a decrease in the diffusion length of surface adatoms. As a result, the surface density of nanoislands increases with increasing their lateral interaction through the elastic deformation fields. This interaction facilitates nanoisland ordering in the growth plane.

The self-ordering effect of nanoislands grown on SiGe buffer layers can be amplified by unintentional miscut within the range of $0.2-0.5^{\circ}$ of commercially available substrates $\mathrm{Si}(001)$. Until recently, the influence of misorientation of the substrate vicinal surface on epitaxial growth was not specially attended [12]. The step edges of atomic layers specify the modulation of non-uniform deformation fields in the deposited buffer layer [13], which, in turn, becomes the pattern for the ordered formation of nanoisland arrays.

In this paper, we investigate the influence of substrate and buffer miscut on the lateral ordering the 
SiGe nanoislands formed on the $\mathrm{Si}_{1-\mathrm{x}} \mathrm{Ge}_{\mathrm{x}}$ sublayer in dependence on the Ge amount. We demonstrate that high homogeneity in distribution of islands can be achieved when depositing 10 monolayers (ML) of Ge. Detailed characterization reveals that the direction of ordering the islands is given by the miscut direction, which has not been observed previously. In particular, we showed that the lateral period of nanoislands depends on the substrate misorienation value.

The samples under investigation were grown using molecular-beam epitaxy on $\mathrm{Si}(001)$ substrates with the controlled miscut $0.3^{\circ}$ at $700{ }^{\circ} \mathrm{C}$. After thermal refinement of substrate in the growth chamber and the growth of 100-nm thick Si buffer layer, the 10-nm thick strained $\mathrm{Si}_{1-\mathrm{x}} \mathrm{Ge}_{\mathrm{x}}$ sublayer with $\mathrm{Ge}$ fraction $x=$ $0.22 \pm 0.02$ was deposited. Self-assembled $\mathrm{Ge}$ nanoislands were formed on $\mathrm{Si}_{1-\mathrm{x}} \mathrm{Ge}_{\mathrm{x}}$ sublayer in Stranski-Krastanov growth mode during deposition of the Ge layer with various thicknesses from 9 to $11 \mathrm{ML}$.

HRXRD measurements were performed with PANalytical X'Pert PRO MRD XL diffractometer. To obtain information about strains and their relaxation during nanoisland ordering, the reflection curves (RCs) and high-resolution reciprocal space maps (HR-RSMs) were measured for symmetrical 004 and asymmetrical 113, 404 reflections [14].

$\mathrm{Si}$ substrate was slightly misoriented in the direction close to $[-100]$, with the miscut components along $[-1-10]$ near $0.4^{\circ}$ and along $[-110]-0.3^{\circ}$. The buffer layer, in its turn, was $0.2^{\circ}$ misoriented only in the [-110] direction.

The layer misorientation increases with increasing the Ge content due to the lattice mismatch. The nature of layer misorientation on miscut substrate is given in [15].

Substrate surface deviations from exact crystallographic orientation are the triggers for the formation of anisotropically ordered system of quantum nanoislands due to different conditions for diffusion processes. Besides, the growth of strained SiGe layer on miscut substrate will cause the periodical strain modulation in the places of breaking the atomic steps [15], which increases the ordering degree (Fig. 1). The unit cells close to each $\mathrm{SiGe} / \mathrm{Si}$ interface will be inhomogeneously strained because of the atomic steps [16].

Modulation of elastic strain fields is caused by the reduction of chemical potential in the places with greater mismatch between $\mathrm{Si}$ substrate and $\mathrm{SiGe}$ layer. The following growth of Ge wetting layer (WL) reaches the critical thickness at a less equivalent amount of deposited Ge. In other words, the nanoislands are formed in the places of elastic strain localization [15]. In the case of vicinal surface of $\mathrm{Si}_{1-\mathrm{x}} \mathrm{Ge}_{\mathrm{x}}$ layer, the nonuniform fields of elastic strains, which could decrease the critical thickness of WL, are absent.

Fig. 2 presents the RC's obtained in asymmetrical diffraction geometry for 113 and 404 reflections measured in $\{110\}$ and $\{100\}$ scattering planes. One can clearly see the system of lateral satellites, confirming the ordering of nanoislands. The distance between lateral satellites allows us to determine the mean distance between nanoislands: from 113 reflections - the distance along [110] direction, from mutually perpendicular (404) scans - along [100] and [010]. These values are given in Table.

Reciprocal space maps measured for asymmetrical 113 and 404 reflections confirmed our suggestions about ordering character in dependence on the thickness of deposited Ge (Fig. 3).

The nanoisland ordering degree increases with $\mathrm{Ge}$ content increasing from 9 to $10 \mathrm{ML}$. This is seen from the appearance of the $2^{\text {nd }}$-order lateral satellites on RCs and HR-RSMs (Fig. 3b). When the Ge layer thickness is $11 \mathrm{ML}$, these satellites disappear because of the bad structural properties [17]. Broadening the diffuse scattering background perpendicular to the diffraction vector can indicate the appearance of chaotically distributed misfit dislocations leading to the structural relaxation (Fig. 3c).

One can observe the additional "streaks" on 113 HR-RSMs close to crystal truncation rod. We suppose that this diffraction effect is the result of mirror reflection of X-rays from island facets aligned along $\{110\}$. In 404 HR-RSMs, we did not observe this effect, since there is no scattering from facet edges. These streaks can be criteria of ordering the planar quantum dots.

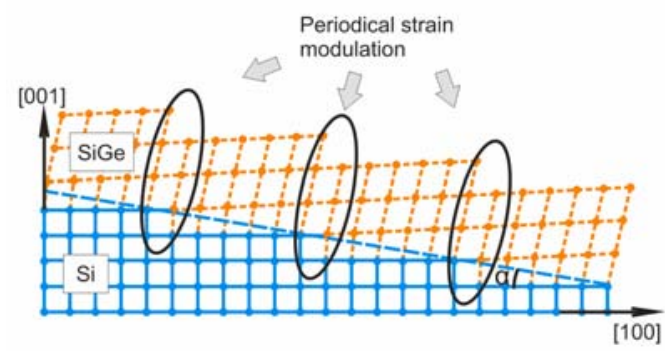

Fig. 1. Periodical strain modulation in the $\mathrm{SiGe} / \mathrm{Si}$ with unintentional miscut.

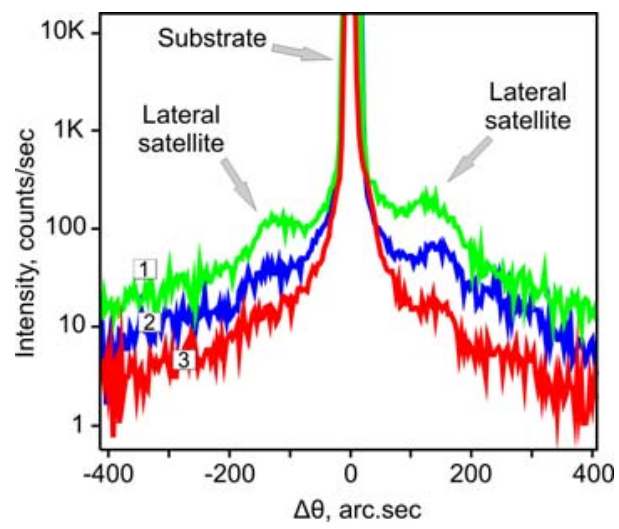

Fig. 2. $\omega$-scans for samples with 9,10 and $11 \mathrm{ML}$ of deposited Ge $(1,2,3)$, respectively. 
Table. Lateral period placement of islands along different crystallographic directions.

\begin{tabular}{|c|c|c|c|c|c|c|}
\hline $\begin{array}{c}\text { Ge } \\
\text { thickness }\end{array}$ & $113(0)$ & $113(90)$ & $404(45)$ & $404(-45)$ & $\begin{array}{c}\text { Sublayer/ } \\
\text { islands, } x\end{array}$ & $\begin{array}{c}\text { Sublayer/ } \\
\text { islands, } \varepsilon_{\mathrm{xx}}\end{array}$ \\
\hline $9 \mathrm{ML}$ & 260 & 269 & 177 & 164 & $0.30 / 0.29$ & $-0.014 /-0.003$ \\
\hline $10 \mathrm{ML}$ & 255 & 246 & 172 & & $0.32 / 0.31$ & $-0.013 /-0.001$ \\
\hline $11 \mathrm{ML}$ & 226 & 226 & 165 & 157 & $0.33 / 0.32$ & $-0.012 / 0.001$ \\
\hline
\end{tabular}
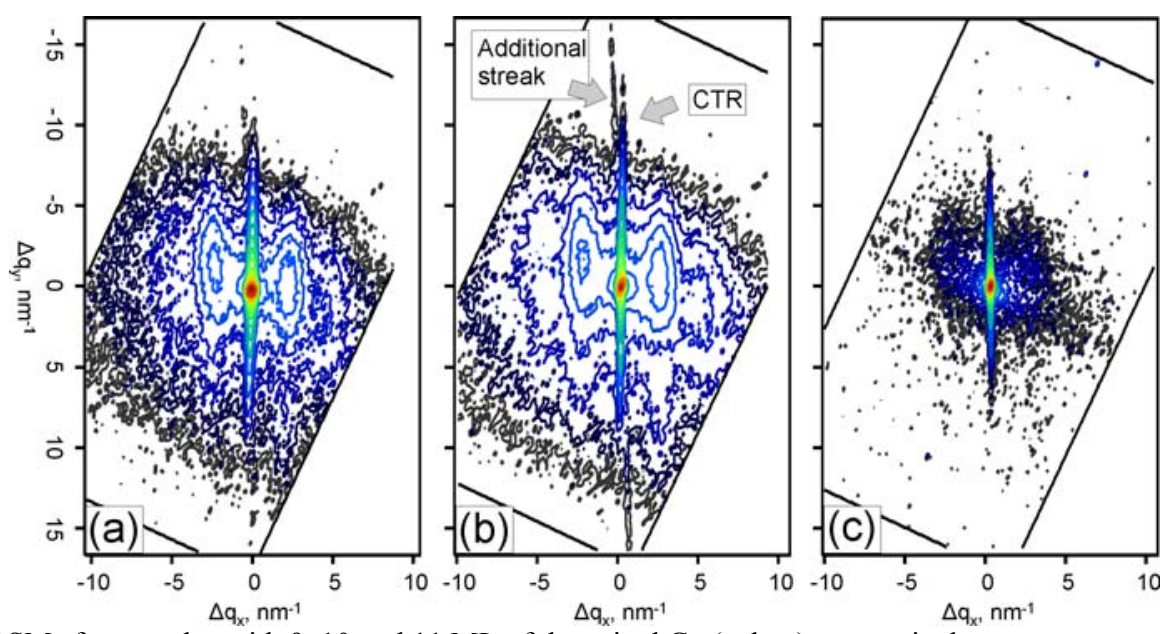

Fig. 3. 113 HR-RSMs for samples with 9,10 and $11 \mathrm{ML}$ of deposited $\mathrm{Ge}$ (a, b, c), respectively.

As it was showed in [18], increase in the deposited Ge nominal thickness from 9 to $10 \mathrm{ML}$ improves the ordering degree, which is seen from the high-order maxima in AFM autocorrelation profiles. A further increase in the Ge thickness up to $11 \mathrm{ML}$ changes the size and shape of nanoislands that appear to be of an elliptical shape. This is a result of anisotropic character of material surface diffusion.

The decrease in the lateral period of nanoislands versus the deposited Ge thickness is evidently given by an increase in their density [18]. The observed difference of lateral period in mutually perpendicular directions for 113 reflections as well as for 404 reflections is a result of the difference of nanoislands planar unit cell from their square shape due to surface diffusion anisotropy. Note that the angle between nanoisland distribution directions was measured to be $\approx 82^{\circ}$. Thus, the planar unit cell representing the two-dimensional arrangement of nanoislands is an oblique lattice.

We have investigated the spatial ordering of nanoislands grown with different thicknesses of deposited Ge layer on the $\mathrm{Si}_{1-\mathrm{x}} \mathrm{Ge}_{\mathrm{x}}$ sublayer. It has been shown that surface-ordered fields of elastic strains initiated by misorientation of substrate and buffer layers amplify the role of interdiffusion processes, which gives the anomalously great flux of atoms from the buffer sublayer into the islands. The ordered character of deformation fields gives rise to the formation of self- ordered nanoisland system. The optimal nanoisland ordering was observed at $10 \mathrm{ML}$ Ge deposition.

The authors acknowledge the financial support of the National Academy of Sciences of Ukraine (projects No 3.5.1.12, No 3.5.1.30) and State Agency for Science, Innovation and Informatization of Ukraine (project $\mathrm{M} / 212-2011$ ).

\section{References}

1. J. Phillips, Evaluation of the fundamental properties of quantum dot infrared detectors // J. Appl. Phys. 91, p. 4590 (2002).

2. V.A. Shchukin, N.N. Ledentsov, D. Bimberg, Epitaxy of Nanostructures. NanoScience and Technology. Springer, 387, 2004.

3. Y. Wakayama, G. Gerth, P. Werner, U. Gosele, L.V. Sokolov, Structural transition of Ge dots induced by submonolayer carbon on Ge wetting layer // Appl. Phys. Lett. 77, p. 2328-2330 (2000).

4. S. Kim, N. Usami, and Y. Shiraki, Selective epitaxial growth of dot structures on patterned $\mathrm{Si}$ substrates by gas source molecular beam epitaxy // Semiconductor Science and Technolology, 14, p. 257-265 (1999).

5. S.Yu. Shiryaev, F. Jensen, J.L. Hansen J.W. Petersen, A.N. Larsen, Nanoscale structuring 
by misfit dislocations in $\mathrm{Si}_{1-\mathrm{x}} \mathrm{Ge}_{\mathrm{x}} / \mathrm{Si}$ epitaxial systems // Phys. Rev. Lett., 78, p. 503-506 (1997).

6. Zhenyang Zhong, A. Halilovic, T. Fromherz, F. Schäffler, and G. Bauer, Two-dimensional periodic positioning of self-assembled $\mathrm{Ge}$ islands on prepatterned Si (001) substrates // Appl. Phys. Lett. 82, p. 4779-4781 (2003).

7. D.N. Lobanov, A.V. Novikov, N.V. Vostokov, Y.N. Drozdov, A.N. Yablonskiy, Z.F. Krasilnik, M. Stoffel, U. Denker, O.G. Schmidt, Growth and photoluminescence of $\mathrm{Ge}(\mathrm{Si})$ self-assembled islands obtained during the deposition of Ge on a strained SiGe layer // Optical Materials, 27, p. 818 (2005).

8. P. Sutter, M.G. Lagally, Nucleationless threedimensional island formation in low-misfit heteroepitaxy // Phys. Rev. Lett. 84, p. 4637-4640 (2000).

9. D.V. Yurasov, Yu.N. Drozdov, The critical thickness of Stranski-Krastanow transition with accounted segregation effect // Semiconductors, 42, p. 563 (2008).

10. D.V. Yurasov, Yu.N. Drozdov, M.V. Shaleev, A.V. Novikov, Features of two-dimensional to three-dimensional growth mode transition of $\mathrm{Ge}$ in $\mathrm{SiGe} / \mathrm{Si}(001)$ heterostructures with strained layers // Appl. Phys. Lett. 95, 151902 (2009).

11. T. Roch, V. Holy, A. Daniel, E. Hoflinger, M. Meduna, T.H. Metzger, G. Bauer, J. Zhu, K. Brunner, G. Abstreiter, X-ray studies on selforganized wires in SiGe/Si multilayers // J. Phys. D: Appl. Phys. 34, A6 (2001).
12. C. Tegenkamp, Vicinal surfaces for functional nanostructures // J. Phys.: Condens. Matter, 21, 013002 (2009).

13. V. Holy, A.A. Darhuber, J. Stangl, G. Bauer, J. Nutzeland, G. Abstreiter, Oblique roughness replication in strained $\mathrm{SiGe} / \mathrm{Si}$ multilayers // Phys. Rev. B, 57, p. 12435 (1998).

14. M. Meduna, V. Holy, T. Roch, G. Bauer, O.G. Schmidt, K. Eberl, X-ray reflectivity from self-assembled structures in $\mathrm{Ge} / \mathrm{Si}$ superlattices // J . Phys. D. Appl. Phys. 34, p. A193 (2001).

15. O. Yefanov, V. Kladko, O. Gudymenko, V. Strelchuk, Yu. Mazur, Zh. Wang, G. Salamo, Fields of deformation anisotropy exploration in multilayered (In,Ga)As/GaAs structures by highresolution X-ray scattering // Phys. Status Solidi (a), 203, p. 154-159 (2006).

16. J. Stangl, V. Holy, G. Bauer, Structural properties of self-organized semiconductor nanostructures // Rev. Modern Phys., 76, p. 725 (2004).

17. V.P. Klad'ko, L.I. Datsenko, J. Bąk-Misiuk, S.I. Olikhovskii, V.F. Machulin, I.V. Prokopenko, V.B. Molodkin, Z.V. Maksimenko, Calculation of two-dimensional maps of diffuse scattering by a real crystal with microdefects and comparison of results obtained from three-crystal diffractometry // J. Phys. D: Appl. Phys., 34, p. A87 (2001).

18. M.Ya. Valakh, P.M. Lytvyn, A.S. Nikolenko, V.V. Strelchuk, Z.F. Krasilnik, D.N. Lobanov, A.V. Novikov, Gigantic uphill diffusion during self-assembled growth of Ge quantum dots on strained SiGe sublayers // Appl. Phys. Lett. 96, 141909 (2010). 